Recepción: 19 / 11 / 2018

Aceptación: 28 / 12 / 2018

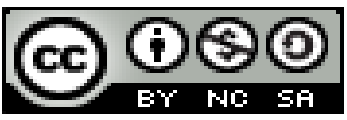

Ciencias de la salud

Publicación: 20 / 02 / 2019

Artículo de Revisión

\title{
Prevención frente a la presencia de problemas dermatológicos del recién nacido
}

\section{Prevention against the presence of dermatological problems of the newborn}

\section{Prevenção contra a presença de problemas dermatológicos do recém-nascido}

Gabriela J. Garcés-Vera ${ }^{\mathrm{I}}$

jggv_92@hotmail.com

Oscar I. Navarrete-Espinoza II

oskrivan25@gmail.com

Olmedo X. Ruiz-Lara III

olxarula@gmail.com

Monica E. Tovar-Gavilanes IV

monicatovar719@yahoo.com

Sarai Elizabeth Del Castillo Pavon V

skally_placebo@hotmail.com

Paulina E. Durán-Mora VI

paupau_edm@hotmail.com

Correspondencia:.jggv_92@hotmail.com

\footnotetext{
Médico General; Hospital Rodríguez Zambrano; Manta, Ecuador. Médico General; Hospital Rodríguez Zambrano; Manta, Ecuador.

Médico General; Residente del Hospital del IESS; Área de Traumatología; Ibarra, Ecuador.

Médico General; Residente del Hospital del IESS; Área de Anestesiología; Ibarra, Ecuador.

Médico General; Residente del Hospital del IESS; Área de Ginecología/ obstetricia; Ibarra, Ecuador.

Medico General; Residente del Hospital General Privado Durán; Área de Emergencia; Ambato, Ecuador.
} 


\title{
Resumen
}

La piel es el órgano con mayor extensión en el cuerpo humano, una de sus principales funciones es proteger, sin embargo, es el más susceptible. Los mecanismos de inmunidad de los recién nacidos, y particularmente de los prematuros, son relativamente deficientes debido a inmadurez del sistema inmune, así como a una disminución del pasaje transplacentario de anticuerpos maternos, todo lo que contribuye a la vulnerabilidad a infecciones graves bacterianas, fúngicas y virales. Debido a la vulnerabilidad del paciente, a las distintas limitantes que se producen al atender el tema e incluso a la ausencia de información para los cuidados necesarios se ha decidido hacer un procesos investigativos a fin de identificar algunos cuidados que hay que mantener en la pile del recién nacido para evitar patologías o complicaciones dermatológicas. Se ha realizado una revisión bibliográfica a través de ficheros virtuales, utilizando palabras claves como dermatología, recién nacido, pediatría, patologías de piel, se ha escogido de estos trabajos la información que nos interesa para exponer una revisión bibliográfica referencial en cuanto a los cuidados necesarios para el cuidado dermatológico de los recién nacidos. Encontrando que la prevención de la sepsis neonatal comienza con la prevención del parto prematuro, se debe dirigir los esfuerzos en colaborar como parte de un equipo multidisciplinario asegurando el cumplimiento de las estrategias de prevención. El cuidado inadecuado de la piel es una causa de morbilidad y aún la mortalidad en ésta primera etapa de la vida. Es importante considerar la trascendencia de éste órgano como barrera contra las infecciones, protección de los órganos internos, colaborador en el mantenimiento de la temperatura y regulador de agua y electrolitos. Reducir al mínimo las lesiones debe ser considerado un componente clave en el cuidado diario brindado a los recién nacidos.

Palabras claves: Piel; Recién Nacido; Neonato; Cuidados; Prevención.

\begin{abstract}
The skin is the organ with the greatest extension in the human body, one of its main functions is to protect, however, it is the most susceptible. The immunity mechanisms of newborns, and those of premature babies, immunity problems, immunity systems, immunity systems, bacterial, fungal diseases. and viral. According to the patient's usefulness, the limitations that are lent to the subject and the absence of information for the care. Newborn to avoid pathologies or dermatological complications. A bibliographic review has been made through the virtual archives, using key words such as dermatology, newborn, pediatrics, skin pathologies, these works have been chosen, the
\end{abstract}


information that interests us for a bibliographic review, reference in the care of the children. For the dermatological care of newborns. Finding that the prevention of neonatal sepsis begins with the prevention of premature birth, efforts should be directed to collaborate as part of a multidisciplinary team ensuring compliance with prevention strategies. Adequate skin care is a cause of morbidity and even more so during the first stage of life. It is important to consider the importance of an organ as a barrier against infections, the protection of internal organs, the collaborator in the maintenance of temperature and the regulator of water and electrolytes. Minimizing injuries should be a key component in the daily care provided to newborns.

Keys words: Skin; Newborn; Newborn; Care; Prevention.

\section{Resumo.}

A pele é o órgão com maior extensão no corpo humano, uma de suas principais funções é proteger, no entanto, é o mais suscetível. Os mecanismos de imunidade de recém-nascidos, e aqueles de bebês prematuros, problemas de imunidade, sistemas de imunidade, sistemas de imunidade, doenças bacterianas, fúngicas. e viral. De acordo com a utilidade do paciente, as limitações que são emprestadas ao sujeito e a ausência de informações para o cuidado. Recém-nascido para evitar patologias ou complicações dermatológicas. Foi realizada uma revisão bibliográfica através dos arquivos virtuais, utilizando palavras-chave como dermatologia, recém-nascidos, pediatria, patologias da pele, estes trabalhos foram escolhidos, a informação que nos interessa para uma revisão bibliográfica, referência no cuidado das crianças. Para o atendimento dermatológico de recém-nascidos. Considerando que a prevenção da sepse neonatal começa com a prevenção do nascimento prematuro, os esforços devem ser direcionados para colaborar como parte de uma equipe multidisciplinar que garanta o cumprimento das estratégias de prevenção. $\mathrm{O}$ cuidado adequado da pele é uma causa de morbidade e ainda mais durante a primeira fase da vida. É importante considerar a importância de um órgão como barreira contra infecções, a proteção de órgãos internos, o colaborador na manutenção da temperatura e o regulador de água e eletrólitos. A minimização de lesões deve ser um componente fundamental no cuidado diário oferecido aos recém-nascidos.

Palavras chaves: Pele; Recém-nascido; Recém-nascido; Cuidado; Prevenção. 


\section{Introducción.}

La piel es el órgano con mayor extensión en el cuerpo humano, una de sus principales funciones es proteger, sin embargo es el más susceptible para observar las patologías porque es muy accesible y por esa evidencia también sirve para mostrar otras patologías internas del cuerpo.

Pocos estudios han sido publicados tratando este tema y poco se ha discutido en eventos científicos. Como ocurre con toda la dermatología neonatal, es considerada como una patología "huérfana" donde los pediatras dicen que es de competencia dermatológica, los dermatólogos dicen que es de competencia neonatológica, y por este motivo no queda bien establecida una figura responsable. (Cutrone \& Peruzzetto, 2006)

La importancia de la piel radica no sólo en su función protectora, sino en su compleja fisiología. Factores como la edad, el sexo, la raza y el clima pueden modificar su aspecto y constitución, alterando su vulnerabilidad. Las enfermedades de la piel constituyen uno de los principales motivos de consulta en la atención primaria. Los niños, en particular los recién nacidos y lactantes, poseen una piel fina, anatómicamente más delgada (la capa córnea no se completa hasta los 4 años de edad) y menos pilosa, en cuanto al $\mathrm{pH}$ local, su escasa acidez y el bajo contenido de ácidos grasos disminuye la resistencia a distintas agresiones por agentes bacterianos, parasitarios y ambientales. Fisiológicamente, la secreción de las glándulas sudoríparas y sebáceas es más escasa, por otro lado, las glándulas apocrinas se encuentran total o parcialmente inactivadas, hasta cerca de la pubertad. Bajo estas circunstancias y debido a que la piel de los niños está expuesta a todo: desde el descuido más absoluto hasta el cuidado excesivo, aunado al hecho de que aún no ha adquirido una inmunidad ideal, sus reacciones suelen ser más aparatosas y en ocasiones más graves que en el adulto. (Roye, Meléndez, Ruíz, Gamboa, \& Morantes, 2006) 
Los mecanismos de inmunidad de los recién nacidos, y particularmente de los prematuros, son relativamente deficientes debido a inmadurez del sistema inmune, así como a una disminución del pasaje transplacentario de anticuerpos maternos, todo lo que contribuye a la vulnerabilidad a infecciones graves bacterianas, fúngicas y virales. El diagnóstico de sepsis neonatal representa un gran desafío ya que su presentación es inespecífica y la utilidad de las pruebas de laboratorio actuales es limitada debido a su baja sensibilidad. El aislamiento del microorganismo a partir de sangre u otros líquidos estériles es considerado el estándar de oro para el diagnóstico de sepsis neonatal. (Herrera, y otros, 2018)

La sepsis es la principal causa de muerte de los pacientes críticamente enfermos en los países en vías de desarrollo. La epidemiología es pobre en las naciones del tercer mundo por lo que se cita con frecuencia datos de países industrializados. La incidencia de infección en países subdesarrollados es de 2.2 a 8.6 por cada mil nacidos vivos; $48 \%$ sucede en los menores de un año y $27 \%$ en el periodo neonatal. Cinco millones de pacientes fallecen en el periodo neonatal anualmente $(98 \%$ en naciones tercermundistas), la mayoría de ellos por infecciones, prematurez y asfixia; las infecciones neonatales provocan alrededor de 1.6 millones de muertes neonatales, en su mayoría debido a sepsis y meningitis. El diagnóstico de infecciones en estancia hospitalaria es entre 33 y $66 \%$ de los recién nacidos ingresados a la Unidad de Cuidados Intensivos Neonatal (UCIN). En América Latina la incidencia de sepsis neonatal se encuentra entre 3.5 y 8.9 por ciento. (Coronell, Pérez, Guerrero, \& Bustamante, 2009)

Debido a la vulnerabilidad del paciente, a las distintas limitantes que se producen al atender el tema e incluso a la ausencia de información para los cuidados necesarios se ha decidido hacer un proceso investigativo a fin de identificar algunos cuidados que hay que mantener en la pile del recién nacido para evitar patologías o complicaciones dermatológicas. 


\section{Metodología.}

Se ha realizado una revisión bibliográfica a través de ficheros virtuales, utilizando palabras claves como dermatología, recién nacido, pediatría, patologías de piel.

El buscador de información virtual nos ha direccionado a libros y artículos científicos que han sido publicados y reconocidos por índices académicos, por tanto, se consideran aceptados y respetados como investigaciones con valides académica que proporcionan información confiable para el ámbito científico.

Se han escogido de estos trabajos la información que nos interesa para exponer una revisión bibliográfica referencial en cuanto a los cuidados necesarios para el cuidado dermatológico de los recién nacidos.

\section{Resultados.}

Como ya conocemos tratar de recopilar informscion acerca de los cuidados dermatológicos de un recién nacido nos direcciona a dos grandes fuentes de información que se pretende unir, la dermatología y la pediatría en el ámbito Neonatal, para ello se delimita principalmente el onjeto de estudio por tanto a manera de conceptualización presentamos un conjunto de definiciones que nos permiten enfocarnos.

El Recién nacido es producto de la concepción desde el nacimiento hasta los 28 días de edad. El Recién nacido vivo, se trata de todo producto de la concepción proveniente de un embarazo de 21 semanas o más de gestación que después de concluir su separación del organismo materno manifiesta algún tipo de vida, tales como movimientos respiratorios, latidos cardiacos o 
movimientos definidos de músculos voluntarios. De acuerdo con la edad de gestación, el recién nacido se clasifica en:

- Recién nacido pretérmino: Producto de la concepción de 28 semanas a menos de 37 semanas de gestación.

- Recién nacido inmaduro: Producto de la concepción de 21 semanas a 27 semanas de gestación o de 500 gramos a menos de 1,000 gramos.

- Recién nacido prematuro: Producto de la concepción de 28 semanas a 37 semanas de gestación, que equivale a un producto de 1,000 gramos a menos de 2,500 gramos.

- Recién nacido a término: Producto de la concepción de 37 semanas a 41 semanas de gestación, equivalente a un producto de 2,500 gramos o más.

- Recién nacido postérmino: Producto de la concepción de 42 semanas o más de gestación.

- Recién nacido con bajo peso: Producto de la concepción con peso corporal al nacimiento menor de 2,500 gramos, independientemente de su edad de gestación.

- Y de acuerdo con el peso corporal al nacer y la edad de gestación los recién nacidos se clasifican como:

1. De bajo peso (hipotrófico): Cuando éste es inferior al percentil 10 de la distribución de los pesos correspondientes para la edad de gestación.

2. De peso adecuado (eutrófico): Cuando el peso corporal se sitúa entre el percentil 10 y 90 de la distribución de los pesos para la edad de gestación.

3. De peso alto (hipertrófico): Cuando el peso corporal sea mayor al percentil 90 de la distribución de los pesos correspondientes a la edad de gestación. (Gómez, Danglot, \& Aceves, 2012) 
La piel constituye un órgano vital muy extenso que con sus múltiples funciones contribuye a asegurar el organismo humano, su vida y su salud, y protege del ambiente a todos los órganos y aparatos del cuerpo. Su importancia no radica sólo en su función protectora, sino en su trabajo fisiológico muy complejo. La edad, el sexo, la raza y el clima son factores que modifican el aspecto y su constitución, y la hacen más o menos vulnerable frente a diferentes enfermedades Por su extensión y por ser el más externo incrementa la posibilidad de ser afectada por enfermedad, trauma y en general agresiones de cualquier naturleza.

En el caso de los niños y específicamente los recién nacidos y lactantes, éstos poseen una piel fina, suave, que está expuesta a todo: desde el descuido más absoluto hasta el cuidado excesivo y ambos pueden igualmente alterar su integridad; por otra parte no ha adquirido aún la inmunidad o la resistencia a las infecciones, picaduras, etcétera, por lo que sus reacciones suelen ser más aparatosas y en ocasiones más graves. Difiere también de la piel del adulto en que es físicamente más permeable a muchas sustancias, sobre todo líquidos que entran en contacto con ella. Anatómicamente es más delgada y las glándulas sudoríparas y sebáceas son menos numerosas. Químicamente se debe destacar el $\mathrm{pH}$ local, cuya escasa acidez debido al ácido láctico y al bajo contenido de ácidos grasos disminuye la resistencia a distintas agresiones por agentes bacterianos y parasitarios. Fisiológicamente el sudor y la secreción de glándulas sebáceas es más escaso. Las glándulas apocrinas se encuentran total o parcialmente inactivadas, hasta cerca de la pubertad. (Martínez, Pastrana, Ramirez, Naranjo, \& Ortiz, 1999)

Las causas dermatológicas suponen un motivo habitual de consulta en Pediatría de Atención Primaria (AP) y reflejan tanto la frecuencia de éstos, como la manifiesta preocupación de los padres por dichos problemas, que afortunadamente no suelen ser graves. Pero esta situación incide tanto en AP como en Atención Hospitalaria, sin olvidar los servicios de urgencias pediátricas, de ahí que el 
pediatra esté necesariamente obligado a incorporarlos a su práctica diaria y a contar con recursos y habilidades para su manejo. Tal aproximación a los problemas dermatológicos requiere conocimientos, actitudes y habilidades adicionales por parte de los profesionales dedicados a la atención pediátrica.

La frecuencia global por procesos dermatológicos observada en AP presenta amplias variaciones en diversos estudios y oscila entre un 5,5 y un 22,5\%. Los bajos porcentajes en algunos casos pueden deberse a causas fundamentales como que los padres manejan generalmente con éxito procesos tan comunes como la costra láctea, la dermatitis del pañal o el acné de sus hijos sin necesidad de consultar por dichos motivos, y acuden a las consultas sólo en los casos en que no obtienen solución al problema. (Menéndez Tuñón, Sariego Jamardo, Fernández Tejada, Fernández García, López Vilar, \& Meana Meana, 2010)

Del libro Dermatología de Jean Bolognia y otros (2018) se extrae el siguiente cuadro que expone algunas necesidades de la piel, esto no se remite exclusivamente a la piel del recién nacido pero nos proporciona una guía de la necesidades del órgano.

Tabla 1: Necesidades y deficiencias selectivas de la piel 
Gabriela J. Garcés-Vera; Oscar I. Navarrete-Espinoza; Olmedo X. Ruiz-Lara; Monica E. Tovar-Gavilanes; Sarai Elizabeth Del Castillo Pavon; Paulina E. Durán-Mora

\begin{tabular}{|l|l|}
\hline $\begin{array}{l}\text { Necesidades } \\
\begin{array}{l}\text { Prevención de la infección a } \\
\text { través de una inmunidad innata } \\
\text { y adaptada }\end{array}\end{array}$ & $\begin{array}{l}\text { Infecciones fúngicas, bacterianas } \\
\text { y víricas; autoinmunidad, cáncer }\end{array}$ \\
\hline $\begin{array}{l}\text { Mantenimiento de barrera } \\
\text { Reparación de lesiones }\end{array}$ & $\begin{array}{l}\text { Infecciones, deshidratación } \\
\text { Cáncer, úlceras en las piernas }\end{array}$ \\
\hline Aporte circulatorio & $\begin{array}{l}\text { Infartos (debidos a embolias, } \\
\text { vasculitis u otras formas de oclusión) }\end{array}$ \\
\hline Comunicación & Neuropatia sensitiva, prurito \\
\hline $\begin{array}{l}\text { Aporte de nutrición } \\
\text { Regulación de la temperatura }\end{array}$ & Deficiencia de vitamina D \\
\hline Llamada de atención & Hipotermia, hipertermia \\
\hline
\end{tabular}

Fuente: (Bolognia, Schaffer, \& Cerroni, 2018)

Factores maternos, ambientales y del huésped determinan que los neonatos expuestos a un microorganismo potencialmente patógeno desarrollarán infecciones severas La inmunidad celular y humoral en los recién nacidos no está completamente desarrollada (la actividad fagocítica, la síntesis de inmunoglobulinas, la actividad del complemento o la función de los linfocitos T2). Durante el período intrauterino no existe ningún estímulo inmunológico significativo que active reacciones inmunitarias preventivas. (Coronell, Pérez, Guerrero, \& Bustamante, 2009)

Durante el primer mes de nacido, el niño es especialmente sensible a las infecciones. Su piel esta cubierta de vermix caseosa, que le proporciona un PH alcalino durante algunos días; es muy fina, estéril y carece de secreciones sudorales y seborreicas así como de flora comenzal. Aunque el proceso de inmunización se inicia rápidamente (en un plazo inferior a 48 horas desde el nacimiento), su progresión es lenta; por ello, el recién nacido está expuesto a un gran riesgo de contaminación. La gran debilidad inmunulógica y la facilidad con que los microorganismos pueden atravesar la barrera 
cutánea, obliga a ser extremadamente prudente con los objetos que entran en contacto con el niño, así como con la higiene de las personas que lo manipulan. (Chattás, 2010)

Históricamente, la prolongación de la estadía hospitalaria ha aumentado el riesgo de contraer una infección intrahospitalaria y una vez adquirida la infección, la estadía se prolonga aún más. El tiempo de contacto con el nosocomio, expone al paciente a la realización de una serie de maniobras invasivas y según las características del servicio donde se encuentra, se hace más susceptible a contraer una infección. Una vez que se adquiere, las complicaciones que pueden presentarse y la utilización de tratamientos antibióticos de larga duración y otros procederes, son entre otras, causas de la prolongación de la estadía hospitalaria del paciente. (Chattás, 2010)

En las gestantes con el antecedente obstétrico de sepsis cérvicovaginal, sus recién nacidos tienen 2,3 veces más probabilidades de presentar sepsis que los restantes niños. La integridad de las membranas constituye una barrera mecánica que impide el ascenso y colonización de los gérmenes dentro de la vagina; al ocurrir una rotura prematura de membrana, es más probable que aparezcan infecciones en el feto. Las sepsis cérvicovaginales aumentan la incidencia de rotura prematura de membrana, pues los gérmenes que colonizan la vagina producen enzimas proteolíticas, que degradan el colágeno de la membrana ovular, y al ascender al líquido amniótico pueden modificarlo y aumentar la incidencia de sepsis neonatal. (Chattás, 2010)

La epidermis, el estrato más superficial de la piel, consta en diversas capas de células con diferencias de función y propiedades. La mayoría de las células de la capa basal, la capa interna de la epidermis, tienen la capacidad de proliferar a capas más superficiales, y a medida que migran de la zona basal a la superficie van perdiendo su capacidad de subdividirse. Finalmente entran en una etapa destructiva, pierden su núcleo y se convierten en fibras situadas en una matriz insoluble. Este 
es un proceso que lleva aproximadamente 25 días. En el feto pasarán a formar el unto sebáceo que lo cubre y lo protege. El estrato córneo, la capa más externa de la epidermis, consiste en un grupo de células muertas unidas por lípidos intracelulares y forman la verdadera barrera de protección de la piel. La piel es la primera barrera que poseen los recién nacidos para hacer frente a factores nocivos y agentes del medio ambiente, para adecuarse a la vida intrauterina segura y protegida del mundo exterior. La maduración del estrato corneo se produce después de las 28 semanas, antes de este tiempo la piel esta pobremente queratinizada. En el recién nacido pretérmino el estrato corneo está constituido por queratinocitos con bajo contenido de agua. Estas características hacen que la piel tenga aspecto gelatinoso, transparente y aumente la permeabilidad de la piel. (Chattás, 2010)

Los protocolos de cuidado de la piel tienen como objetivo generar criterios basados en la evidencia en las Unidades de Cuidados Intensivos Neonatal (UCIN), estableciendo como metas principales mantener la integridad cutánea y prevenir las lesiones de la piel. Son objetivos más específicos de un protocolo.

- Valorar la condición de la piel del recién nacido.

- Identificar recién nacidos que se encuentren en riesgo de presentar alteraciones en la integridad de la piel.

- Reconocer tratamientos y agentes que pueden alterar la integridad de la piel.

- Implementar intervenciones para promover y proteger la piel.

- Promover el desarrollo normal de la piel. (Chattás, 2010)

\section{Valoración de la piel del recién nacido}

Para valorar la piel del recién nacido diariamente es adecuado utilizar herramientas objetivas para evitar impresiones personales, con el fin de detectar anormalidades y problemas en la piel de los 
recién nacidos. La Escala de Valoración de la Piel del Recién Nacido (NSCS Neonatal Skin Condition Scale) es un sistema de evaluación que consiste en una escala de 3 ítems que evalúa hidratación, eritema y lesión de la piel. Cada ídem es calificado con puntaje de 1 a 3 . Con un total de 9 puntos la piel se encuentra en riesgo, mientras que si el puntaje es de 3 es el puntaje óptimo en relación al estado de la piel del recién nacido. Existe evidencia de validez y confiabilidad del uso de esta escala (Chattás, 2010)

\section{Tabla 2: Escala de Valoración de la piel del recién nacidos) NSCS; Neonatal Skin Condition Scale)}

\begin{tabular}{|c|c|}
\hline \multicolumn{2}{|c|}{ Hidratación de la piel } \\
\hline $\begin{array}{l}\text { 1- Normal } \\
\text { 2- Piel Seca } \\
\text { 3- Piel Muy Seca }\end{array}$ & $\begin{array}{l}\text { No hay signos de deshidratación } \\
\text { Visiblemente Escaldada } \\
\text { Grietas / Fisuras visibles }\end{array}$ \\
\hline \multicolumn{2}{|r|}{ Eritema } \\
\hline \multicolumn{2}{|c|}{$\begin{array}{l}\text { 1- No hay presencia de eritema } \\
\text { 2- Eritema Visible }<50 \% \text { de la superficie corporal } \\
\text { 3- Eritema Visible }>50 \% \text { de la superficie corporal }\end{array}$} \\
\hline \multicolumn{2}{|r|}{ Eritema } \\
\hline $\begin{array}{l}1=\text { Ninguna } \\
2=\text { Pequeñas áre } \\
3=\text { Áreas extens }\end{array}$ & lizadas \\
\hline
\end{tabular}

Fuente: (Chattás, 2010)

Las siguientes prácticas han demostrado ser efectivas para disminuir las pérdidas transepidérmicas de agua y mantener la hidratación y la integridad de la piel:

- En los recién nacidos menores a 30 semanas, en la recepción del recién nacido, utilizar bolsas de polietileno que cubran cuerpo y extremidades sin realizar el secado por arrastre, que demás lesiona la epidermis.

- Utilizar sábanas plásticas durante la estabilización, luego del nacimiento. Las mismas reducen las pérdidas por evaporación. Retirar luego de la admisión a la UCIN.13-15

- Movilizar al recién nacido precozmente de servocunas a incubadoras de doble pared. 
- Proporcionar humedad relativa dentro de las incubadora.

Si bien no hay consenso generalizado, las recomendaciones sobre niveles de humedad de la Association of Women`s Health, Obstetric and Neonatal Nurses establece que se administre alta humidificación entre $70 \%$ y $90 \%$ durante los primeros siete días teniendo en cuenta la edad gestacional del recién nacido (23-26 semanas, 85\%, 27-30 semanas, 70-75\%) y luego de la primera semana disminuir gradualmente hasta $50 \%$ hasta los 28 días de vida o al momento de retirarla. Los recién nacidos prematuros mayores de 30 semanas no requieren humedad en la incubadora.

- Utilizar sábanas plásticas o carpas para reducir las pérdidas transepidérmicas de agua. El material plástico no debe estar en contacto directo sobre la piel del recién nacido por periodos prolongados.

- Usar sistemas servocontrolados de humidificación en las incubadoras

- Usar una técnica o la combinación de varias para disminuir la pérdida de agua y minimizar las pérdidas de calor por evaporación en menores de 30 semanas de gestación. Uso de lubricantes y emolientes La hidratación cutánea neonatal está disminuida por actividad deficitaria de las glándulas sudoríparas, disminución de las pérdidas de agua y descenso del agua extracelular. El grado de hidratación del estrato córneo es directamente proporcional a la habilidad de este tejido para mantener y evitar las pérdidas de agua. (Chattás, 2010)

\section{Baño del recién nacido pretérmino}

El baño a los recién nacidos pretérmino tiene muchos efectos potencialmente beneficiosos, pero no es un procedimiento inocuo. El primer baño del recién nacido tiene múltiples propósitos, remover sangre y fluidos potencialmente infecciosos, reducir la colonización microbiana, permitir una 
correcta observación de la piel del recién nacido, además de cuestiones de estética. El baño inicial en la sala de partos es una rutina postergada, debido a que hay rutinas prioritarias de reanimación y estabilización y que realizada antes de las 2-4 horas de vida produce alteraciones en la termorregulación y en los signos vitales. Para el primer baño, en los recién nacidos menores de 32 semanas se utilizará agua estéril templada, si las condiciones clínicas lo permiten. El baño de rutina tiene como objetivo remover suciedad y microorganismos, pero puede dañar la piel neonatal debido a la fragilidad de la epidermis y permitir una mayor colonización de bacterias del medio ambiente adquirido. La mayoría de autores afirman que todos los jabones son irritantes y hacen hincapié en que su uso frecuente es perjudicial, ya que quitan la película de lípidos de la superficie de la piel.

Fisiológicamente, el pH de la piel es neutral al nacer, y se acidifica durante la primera semana de vida, con valores de $\mathrm{pH}$ entre 5,0 a 5,5.24 Este "manto ácido" disminuye la colonización bacteriana y promueve la retención de humedad en la barrera de la piel. Bañar frecuentemente a los recién nacidos pretérmino puede modificar el "manto ácido" y elevar el pH de la piel. Los mecanismos implicados en la colonización de la piel de los recién nacidos prematuros en una UCIN no son totalmente conocidos. La colonización de la piel de un recién nacido en una UCIN es consecuencia de múltiples factores, incluyendo el baño de rutina. No existe ningún diferencia entre bañar con agua o bañar con jabón suave de pH neutro y agua en la flora microbiana de la piel de los recién nacidos prematuros. El uso de antisépticos de rutina está desaconsejado, ya que si bien reducen la colonización, su acción es corta, y producen irritación y resecamiento cutáneo. Se recomienda el uso de jabones líquidos o en barra que tengan un Ph neutro, para que ésta práctica no altere el $\mathrm{Ph}$ de la piel. Se puede alternar baños con agua tibia solamente, con baños con jabón. (Chattás, 2010)

En los recién nacidos menores de 32 semanas se utilizará solo agua tibia durante la primera semana de vida, y en aquellos pacientes que tengan la piel lesionada se utilizará agua estéril para este 
procedimiento. Esta desaconsejado frotar la piel, sino realizar la higiene con torundas de algodón. Los beneficios del baño diario no están bien documentados. La frecuencia del baño en el periodo neonatal se debe basar en las necesidades individuales de cada recién nacido y teniendo en cuenta los valores y creencias de la familia. (Chattás, 2010)

Se denomina baño de inmersión, al baño del recién nacido donde se sumerge el cuerpo dentro de una bañadera excepto cuello y cabeza. Este tipo de baño es considerado seguro y efectivo para mantener la higiene del recién nacido. Recibirán baño de inmersión los recién nacidos prematuros que se encuentren estables, que no tengan catéteres arteriales y que tengan puesto el clamp de cordón. Se evaluará la tolerancia al procedimiento, observando en el recién nacido alteraciones en la frecuencia cardiaca, frecuencia respiratoria y cambios en la saturación, antes, durante y posterior al baño. La cantidad de agua a utilizar será de $12 \mathrm{~cm}$, suficiente agua que cubra todo el cuerpo del recién nacido pretérmino, excepto cabeza y cuello para evitar las perdidas por evaporación. La temperatura del agua deberá ser de 38-40 grados centígrados. La temperatura ambiental debe ser de 26-27 C. Si el recién nacido se encuentra en incubadora el baño debe hacerse dentro de la misma, elevando la temperatura del aire y precalentando las compresas para el secado. Luego del baño, secar al recién nacido cuidadosamente, evitando frotar o restregar la piel, ya que podría lesionar la epidermis. Vestir, cubrir su cabeza y arropar si el estado clínico lo permite. Lavar la bañadera (Chattás, 2010)

\section{Prevención de infección nosocomial}

\section{Lavado de manos}

Es la medida que mejores resultados ha mostrado para disminuir las infecciones nosocomiales, sin embargo, su práctica en el mundo es complicada. En los países subdesarrollados la falta de agua, 
jabón y lavamanos adecuados son los principales inconvenientes, así como personal de enfermería insuficiente y el sobrecupo en las UCIN no favorecen su aplicabilidad; se requieren estrategias como educación continua y retroalimentación para crear conciencia de su necesidad en el personal médico, paramédico y familiares de los pacientes. Una de las estrategias para aumentar la adherencia al lavado de manos es el uso de alcohol glicerinado. (Chattás, 2010)

\section{Nutrición}

La sepsis neonatal se correlaciona con el uso de nutrición parenteral, especialmente cuando contiene lípidos intravenosos, se debe limitar el uso de estas soluciones, así como la permanencia de catéteres umbilicales por el tiempo mínimo posible; iniciar la alimentación enteral lo más pronto posible en los neonatos, si es pretérmino se comienza con dosis tróficas de leche idealmente materna. En general el uso de leche materna de forma precoz disminuye el riesgo de enterocolitis necrotizante así como infección nosocomial; la suplementación con glutamina se ha relacionado con una reducción de sepsis nosocomial. (Chattás, 2010)

\section{Catéteres intravasculares}

Existe clara relación entre infección nosocomial y el uso de catéteres centrales; es un factor de riesgo para infecciones por Staphylococcus en especial los coagulasa- negativos. Dentro de las medidas para prevenir su aparición se encuentra la utilización de técnica aséptica para su colocación que incluye: utilización de gorros, tapabocas y guantes estériles. Se debe remover de manera pronta cuando no sea necesario su uso, o cuando haya cultivos repetidos del catéter positivos para Staphylococcus aureus o coagulasas negativos. Se debe utilizar como antiséptico soluciones yodadas o clorhexidina, no se deben utilizar soluciones orgánicas, ni ungüentos antibióticos. El uso de vancomicina profiláctica no se recomienda. El sistema debe estar cerrado, los cambios en este deben 
hacerse con técnica estéril. No se recomienda ordenar cultivos de “punta del catéter” ya que según datos suministrados por el CDC de Atlanta, entre 20 y $25 \%$ están colonizadas. Por último se recomienda crear equipos o comités para la colocación y cuidados del catéter, integrado por enfermeras, personal del comité de infecciones, liderado por el neonatólogo; dentro de sus funciones se encuentran llevar a cabo educación continua, revisión de las guías y protocolos periódicamente, calificar la colocación y los cuidados de estos dispositivos. (Chattás, 2010)

\section{Cuidados de la piel}

La piel de los recién nacidos es frágil, en especial la de los prematuros; en las UCIN debido a los procedimientos invasivos y las tomas de sangre continuas se produce una puerta de entrada para gérmenes intrahospitalarios y riesgo de sepsis neonatal. Un estudio clínico realizado con neonatos de 501 a 1000 g confirmó que la utilización de ungüentos emolientes se relacionó con infecciones nosocomiales. Campbell y colaboradores encontraron que la utilización de petrolato tópico aumentaba el riesgo de candidiasis sistémica. No se recomienda la utilización profiláctica de emolientes. (Chattás, 2010)

\section{Profilaxis antimicrobiana para Streptococcus agalactiae}

El CDC modificó en 2002 su normativa y propuso aplicar sólo el protocolo basado en el cultivo universal a las madres gestantes. Las recomendaciones emitidas son las siguientes:

Cultivo vaginal y rectal selectivo para indentificar S. agalactiae a las 35-37 semanas a toda mujer embarazada; excepto que la paciente presentara bacteriuria por S. agalactiae en el embarazo actual o que existiera el antecedente de enfermedad invasora por S. agalactiae en un hijo previo.

Se indica profilaxis antimicrobiana intraparto si existe: 
- Bacteriuria por S. agalactiae en el embarazo actual.

- Antecedentes de enfermedad invasora por S. agalactiae en un hijo previo.

- Resultado positivo del tamizaje en el embarazo actual (excepto si el parto se resolverá por cesarea electiva y en presencia de membranas integras).

Si el resultado del tamizaje resulta desconocido se administra profilaxis si:

- El trabajo de parto se desencadenó antes de las 37 semanas de gestación.

- Hay ruptura de membranaspor un periodo mayoro igual a 18 horas.

- Se presenta fiebre mayor o igual a $38^{\circ} \mathrm{C}$ intraparto.

- Profilaxis intraparto.

De elección: penicilina G sódica 5.000.000 UI, endovenosa. Repetir 2.500.000 UI, endovenosa, cada 4 horas hasta que se produzca el parto.

De segunda elección: ampicilina de 2 g endovenosa. Luego 1 g endovenosa cada 4 horas hasta que se produzca el parto. En caso de alergia a la penicilina y derivados: cefazolina/cefalotina $2 \mathrm{~g}$ endovenosa, luego $1 \mathrm{~g}$ cada 8 horas hasta el parto, o bien, clindamicina 900mg endovenosa cada 8 horas hasta el parto. En caso de alergia mayor a la penicilina y derivados. Clindamicina igual al esquema anterior. En caso de resistencia del EGB a la clindamicina: vancomicina 1g endovenosa cada 12 horas hasta el parto. Es importante mencionar que el uso intraparto de antibióticos ha reducido la prevalencia de sepsis neonatal temprana por EGB en los países que lo han implementado de forma adecuada, sin embargo, es preocupante que paralelamente ha aumentado la incidencia de 
infecciones por E. coli y K. pneumoniae resistente a la ampicilina. (Coronell, Pérez, Guerrero, \& Bustamante, 2009)

El manejo integral de sepsis neonatal en UCIN incluye:

- Oxigenoterapia.

- Ventilacion Mecánica.

- Liquidos Endovenosos.

- Sopofrte inotrópico.

- Alimentacion parental. (Coronell, Pérez, Guerrero, \& Bustamante, 2009)

Como consecuencia de la inmadurez de la piel neonatal un número de diferentes tipos de heridas se producen en este grupo de pacientes. Las siguientes tablas ofrecen detalles de los diferentes tipos de heridas, la causa de la lesión y proporcionar orientación para evitar la ocurrencia. (Chattás, 2010)

\section{Lesiones Epidermicas}

\begin{tabular}{|l|l|}
\hline Causas & Intervenciones / Medidas de prevención \\
\hline Manipulación del recién nacido. & $\begin{array}{l}\text { Manejar con cuidado extremo al recién nacido } \\
\text { prematuro, los anillos en las manos pueden } \\
\text { causar daño epidérmico. }\end{array}$ \\
\hline $\begin{array}{l}\text { Remoción de telas adhesivas utilizadas para } \\
\text { fijar vías, tubos y drenajes. }\end{array}$ & $\begin{array}{l}\text { Evitar los productos que se adhieran } \\
\text { firmemente a la piel. } \\
\text { Fijar las vías endovenosas con apósitos } \\
\text { transparentes que permita el seguimiento del } \\
\text { sitio, y evitar el cambio frecuente de los }\end{array}$ \\
\hline
\end{tabular}




\begin{tabular}{|l|l|}
\hline & $\begin{array}{l}\text { mismos. } \\
\text { Para la remoción de adhesivos y electrodos, } \\
\text { utilizar solo agua tibia, evitando solventes u } \\
\text { otros productos que se puedan absorber, } \\
\text { teniendo en cuenta la gran permeabilidad que } \\
\text { tiene la piel del recién nacido. Retirar la tela } \\
\text { adhesiva en forma paralela a la piel }\end{array}$ \\
\hline Prevenir las lesiones epidérmicas & $\begin{array}{l}\text { Para prevenir las lesiones epidérmicas se } \\
\text { pueden utilizar elementos para que eviten la } \\
\text { presión sobre los tejidos colchones de agua, } \\
\text { gel o aire, pieles de cordero, apósitos } \\
\text { transparentes o hidrocoloides en la zona de } \\
\text { apoyo: rodillas y codos }\end{array}$ \\
\hline
\end{tabular}

\section{Escorlaciones}

\begin{tabular}{|l|l|}
\hline Causas & Intervenciones / Medidas de prevención \\
\hline $\begin{array}{l}\text { Diarrea, secreciones pulmonares, heces } \\
\text { periostoma y orina sobre la piel. }\end{array}$ & $\begin{array}{l}\text { Cambiar los pañales con frecuencia cada 4-6 } \\
\text { horas. Si el recién nacido se encuentra } \\
\text { inestable es necesario realizar las mínimas } \\
\text { intervenciones y la frecuencia de la } \\
\text { manipulación dependerá de la política de la } \\
\text { UCIN. } \\
\text { Limpiar los genitales sólo con agua, las } \\
\text { toallitas perfumadas son innecesarios. Evitar el } \\
\text { uso de jabones alcalinos o soluciones } \\
\text { jabonosas para el baño del bebé perfumadas } \\
\text { que modifiquen el Ph de la piel. }\end{array}$ \\
$\begin{array}{l}\text { El uso de una pasta de hidrocoloide y pasta de } \\
\text { zinc a veces es una barrera eficaz cuando hay }\end{array}$ \\
\hline
\end{tabular}




\begin{tabular}{|l|l|}
\hline & $\begin{array}{l}\text { pérdidas extraordinarias. Las películas que } \\
\text { producen algunos productos para mejorar la } \\
\text { función de barrera de la piel pueden utilizarse } \\
\text { para la protección, pero deben evaluarse la } \\
\text { absorción del producto y sus posibles } \\
\text { consecuencias. Utilizar productos evaluados } \\
\text { en recién nacidos }\end{array}$ \\
\hline Lesiones producidas por cándida & $\begin{array}{l}\text { Si se sospecha de cándida, es necesario } \\
\text { realizar un cultivo y tratar al mismo tiempo. } \\
\text { Dependiendo del cultivo si es negativo el } \\
\text { tratamiento debe ser interrumpido. }\end{array}$ \\
\hline
\end{tabular}

\section{Quemaduras}

\begin{tabular}{|l|l|}
\hline Causas & Intervenciones / Medidas de prevención \\
\hline $\begin{array}{l}\text { Contacto con Soluciones antisépticas para la } \\
\text { preparación de la piel de base alcohólica } \\
\text { (clorhexidina y yodo). }\end{array}$ & $\begin{array}{l}\text { Las soluciones para la antisepsia de la piel } \\
\text { deben ser en base acuosa y la exposición a las } \\
\text { mismas limitada en el tiempo. }\end{array}$ \\
$\begin{array}{l}\text { Evitar topicar zonas demasiados extensas para } \\
\text { la preparación de la piel durante la inserción } \\
\text { de vías, y otros dispositivos para evitar la } \\
\text { absorción de la misma o que el recién nacido } \\
\text { permanezca acostado en la sábanas mojadas. }\end{array}$ \\
$\begin{array}{l}\text { Retirar antisépticos y cualquier agente de } \\
\text { limpieza inmediatamente con agua estéril } \\
\text { luego de realizar la antisepsia al colocar vías y } \\
\text { otros procedimientos }\end{array}$ \\
$\begin{array}{l}\text { Evitar el uso de soluciones yododas que puede } \\
\text { causar hipotiroidismo y quemaduras graves. }\end{array}$ \\
\hline
\end{tabular}

\section{Quemaduras por calor}

\begin{tabular}{|l|l|}
\hline \multicolumn{1}{|c|}{ Causas } & Intervenciones / Medidas de prevención \\
\hline $\begin{array}{l}\text { Contacto con luces calientes de luminoterapia, } \\
\text { servocunas, e incubadoras en modo servo. }\end{array}$ & $\begin{array}{l}\text { Valorar a los recién nacidos expuestas a estas } \\
\text { terapéuticas en forma frecuente. }\end{array}$ \\
\hline
\end{tabular}




\begin{tabular}{|l|l|}
\hline & $\begin{array}{l}\text { Evitar el uso de servocunas, preferir el uso de } \\
\text { incubadoras ya que aumentan las pérdidas } \\
\text { insensibles y al brindar calor por radiación } \\
\text { favorece a las quemaduras. } \\
\text { Fijar sensores correctamente evitando su salida } \\
\text { accidental. }\end{array}$ \\
\hline $\begin{array}{l}\text { Uso de luces calientes para observación el } \\
\text { recorrido de las venas para facilitar la la } \\
\text { inserción de vías endovenosas o para realizar } \\
\text { procedimientos delicados. }\end{array}$ & $\begin{array}{l}\text { Preferir el uso de luces frías de luminoterapia } \\
\text { para recién nacidos pretérmino } \\
\text { Mantener la distancia correspondiente de } \\
\text { acuerdo al tipo de luminoterapia para evitar } \\
\text { quemaduras especialmente con luz halógena. }\end{array}$ \\
& $\begin{array}{l}\text { Limitar el tiempo de exposición de luces } \\
\text { calientes }\end{array}$ \\
\hline
\end{tabular}

\section{Lesiones por presión:}

La aparición de úlceras por presión es poco frecuente en los recién nacidos prematuros, debido a la gran superficie en relación al peso. Los recién nacidos que son sedados, paralizados y aquellos con presión arterial baja, con administración de inotrópicos, están en mayor riesgo de presentarlas, al igual que los neonatos con edemas. Las úlceras por presión pueden aparecer en las orejas, en el occipucio, si el recién nacido está en decúbito supino, o en las rodillas si se encuentra en decúbito prono. Siempre que sea posible hay que reposicionar de los bebés para prevenir las lesiones y utilizar superficies que alivian la presión. Estos incluyen almohadillas de gel, colchones de aire que redistribuyan la presión. (Chattás, 2010)

\begin{tabular}{|l|l|}
\hline Causas & Intervenciones / Medidas de prevención \\
\hline Sondas. & Evitar que el recién nacido se encuentre \\
& acostado sobre tubos, sondas o cables de \\
\hline
\end{tabular}


Gabriela J. Garcés-Vera; Oscar I. Navarrete-Espinoza; Olmedo X. Ruiz-Lara; Monica E. Tovar-Gavilanes; Sarai Elizabeth Del Castillo Pavon; Paulina E. Durán-Mora

\begin{tabular}{|c|c|}
\hline & monitores. \\
\hline Sensores de saturación y temperatura. & $\begin{array}{l}\text { Rotar sensores cada 3-4 horas o más a menudo } \\
\text { si es necesario para los bebés muy prematuros. }\end{array}$ \\
\hline $\begin{array}{l}\text { Tabique nasal en recién nacidos con presión } \\
\text { positiva continua o Asistencia respiratoria } \\
\text { mecánica. }\end{array}$ & $\begin{array}{l}\text { Considerar el uso de protección con } \\
\text { hidrocoloide en las alas de la nariz y en la zona } \\
\text { peri tubos endotraqueal. }\end{array}$ \\
\hline Recién nacidos edematosos & $\begin{array}{l}\text { Afloje las cintas adhesivas y ropa cuando el } \\
\text { bebé está edematoso. }\end{array}$ \\
\hline
\end{tabular}

\section{Lesiones por extravasación}

\begin{tabular}{|l|l|}
\hline Causas & $\begin{array}{l}\text { Intervenciones / Medidas de prevención } \\
\begin{array}{l}\text { Infusión de soluciones irritantes o o } \\
\text { hiperosmolares. }\end{array}\end{array}$ \\
$\begin{array}{l}\text { Controlar frecuentemente los sitios de } \\
\text { inserción de los catéteres cortos y utilizar vías } \\
\text { entrales cuando la concentración de glucosa } \\
\text { sea mayor a 10 \% Identificarlas } \\
\text { adecuadamente. } \\
\text { Evitar colocar catéteres en lugares con } \\
\text { dificultades en la inmovilización, } \\
\text { especialmente en zonas de flexión o cercanas a } \\
\text { zonas donde se encuentran tendones, nervios o } \\
\text { arterias. }\end{array}$ \\
\hline
\end{tabular}




\begin{tabular}{|l|l|} 
Obstrucción causada por fibrina o trombos & $\begin{array}{l}\text { Fijar las vías con apósitos transparentes que } \\
\text { permiten la buena observación del tejido } \\
\text { circundante. }\end{array}$ \\
Vías de larga duración & $\begin{array}{l}\text { Inspecionar las vías con mucha frecuencia } \\
\text { para detectar signos de extravasación y evitar } \\
\text { lesiones. }\end{array}$ \\
$\begin{array}{l}\text { La duración de las cánulas endovenosas en } \\
\text { recién nacidos es de 36 horas.36 } \\
\text { Ante la presencia de signos de infiltración, } \\
\text { suspender la infusión inmediatamente. Los } \\
\text { síntomas de la infiltración incluyen edema, } \\
\text { dolor en el sitio, calor de la piel, rubor, y en } \\
\text { algunos casos, la falta de retorno de sangre al } \\
\text { catéter. Comunicar a la brevedad y adoptar una } \\
\text { conducta activa dependiendo la droga que se } \\
\text { ha extravasado Se realizarán intervenciones de } \\
\text { enfermería no farmacológicas entre las cuales } \\
\text { la más frecuente es elevar el sitio donde se } \\
\text { encuentra la infiltración o la extremidad } \\
\text { afectada. }\end{array}$ \\
$\begin{array}{l}\text { Se utilizará Nitroglicerina al 2\%, en crema, a } \\
\text { una dosis de } 4 \text { mm/kg ante la isquemia severa } \\
\text { de la piel, en recién nacidos mayores de 21 } \\
\text { días, con piel intacta. El uso de otros agentes } \\
\text { está discutido. }\end{array}$
\end{tabular}

Fuente: Chattás, 2010

El tratamiento de la piel lesionada se realizará con algunos de los siguientes métodos: Las lesiones de la piel se recomienda lavarlas con agua destilada y cubrirlas con parches oclusivos. Los productos que generan un ambiente húmedo ejercen en general, una absorción y retención del exudado, controlando la cantidad del mismo entre el apósito y la lesión. Están constituidos por sustancias con gran afinidad por el agua, que junto con el exudado de la lesión, mantienen un 
ambiente húmedo que favorece la cicatrización. Además los apósitos hidrocoloides producen los siguientes efectos:

- Aumento del aporte de oxígeno y nutrientes a través de la angiogénesis.

- Acidificación del ph de la zona, creando ambiente bacteriostático que disminuye riesgo infección.

- Facilidad para la migración celular, polimorfonucleares y macrófagos, como las de la reparación plástica.

- Control del exudado sin perjudicar la piel lesionada.

- Mantenimiento de la temperatura adecuada, estimulando con ello la fibrinolisis.

- Disminución del dolor.

- Protección a las heridas de la contaminación.

- Reducción de los tiempos de cicatrización.

- Disminución en el número de curas locales.

Los apósitos transparentes adhesivos, y los parches hidrocoloides deben ser usados con cuidado en superficies lesionadas muy extensas. El manejo del dolor debe ser considerada un componente importante durante la curación y tratamiento de las lesiones de todos los recién nacidos, independientemente de su edad gestacional o la gravedad de la enfermedad. La valoración del dolor se ve obstaculizada por la falta de sensibilización por parte de profesionales de la salud que los 
recién nacidos sienten dolor, en parte, debido al hecho de que no puede mostrar una respuesta de comportamiento vigoroso para el dolor y la ansiedad sobre la posibles efectos adversos de analgesia.

\section{Procedimiento para la curación de lesiones}

La curación de lesiones y heridas es un trabajo de equipo. Lo ideal sería realizar este procedimiento entre dos enfermeras, una para la contención y succión no nutritiva para la comodidad del bebé y la otra para hacer la técnica.

Reducir los cambios de apósito a un mínimo, sólo quitar las cintas o vendajes si es esencial. Evitar el ruido, la iluminación brillante y la manipulación para reducir el estrés al bebé. Si la herida requiere de lavado, utilizar líquidos tibios y no fríos ya que pueden causar trauma y mayor dolor. Para poder evaluar la evolución de la lesión, se pueden sacar las fotografías durante la curación, para evitar cambios adicionales cuando algún profesional desee ver la herida.

Los padres pueden sentirse culpables o ansiosos y necesitan adquirir seguridad. Identificar su nivel de comprensión y que quieran saber sobre las lesiones de su bebé. Permitir a los padres a participar en el procedimiento si así lo desean. (Chattás, 2010)

\section{Registros}

Es importante documentar aspectos del cuidado de las lesiones tales como:

- El tipo de lesión.

- Ubicación de la lesión 
- Dimensiones, longitud, ancho y profundidad.

- Naturaleza de la lesión

- Condición de la piel circundante.

- Características del exudado, color y consistencia si lo tuviere

- Presencia de malos olores.

- Presencia de infección.

- Nivel de dolor.

- Alergias o sensibilidad de la piel.

- Tratamiento aplicado (Chattás, 2010)

\section{Cuidados de enfermería profilácticos en las infecciones de los $R \mathrm{~N}$ bajo peso}

- Colocar al recién nacido en una incubadora para proporcionarle un ambiente térmico neutro y a aislarlo del medio ambiente.

- Uso de ropa estéril.

- Manipular al recién nacido lo menos posible, de ser necesario con guantes y sobrebatas estériles.

- Lavado de mano vigoroso, antes de cada manipulación.

- Cambiar los depósitos de agua de las incubadoras cada 24 h y añadirle ácido acético al $2 \%$. 
- Realizar cambios de incubadora cada 7 días.

- Desinfección diaria de la incubadora con agua estéril por las paredes internas y alcohol al 76 $\%$ por la parte externa.

- Relación enfermera-paciente no mayor 1:2.

- Los dispositivos capaces de producir lesiones de la piel (electrodo de cardiomonitores, colectores de orinas, entre otros) sólo se usarán el tiempo estrictamente necesario.

- Evitar la humedad dentro de la incubadora, pues favorece a la proliferación de gérmenes. (Chattás, 2010)

\section{Cuidados de enfermería profilácticos de las infecciones con los catéteres}

- El material de proceder debe estar estéril.

- Extremar las medidas de asepsia y antisepsia durante la realización del proceder.

- Realizar desinfección mecánica (agua y jabón) y química (alcohol al 76 \% y yodo povidona) de la zona antes de la punción.

- Acortar el tiempo de permanencia y que sea el estrictamente necesario.

- No extender la estadía del catéter más allá de lo indicado, catéter umbilical (máximo 7 días), catéter epicutáneo (máximo 21 días).

- Lavado de mano vigoroso, antes de la manipulación.

- Uso de guantes estériles cada vez que se va a manipular el catéter. 
- Proteger la conexión y la llave de 3 pasos con torundas o apósitos estériles.

- Tomar muestra rutinaria de los catéteres.

- Vigilar los signos de infección del área canalizada (rubor, calor, inflamación, flebitis), en caso de existir, retirarlo inmediatamente.

- Evitar el reflujo de sangre por el catéter, en caso de refluir sangre, lavar con solución salina o dextrosa al $5 \%$.

- Evitar que la entrada de los catéteres hagan contacto con áreas no estériles. (Hernandez \& García, 2006)

\section{Cuidados de enfermería profilácticos de la infección durante la ventilación}

- Acortar el tiempo de permanencia según las posibilidades del neonato.

- Aspirar cuando sea necesario y en un tiempo breve.

- Control bacteriológico frecuente de las secreciones y conexiones.

- Cambios de conexiones del equipo y los depósitos de agua del humectador cada 24 h.

- Evitar la presencia de agua en los circuitos del recién nacido, pues favorece la proliferación de gérmenes.

- La manipulación de las conexiones deben ser con guantes estériles.

- Lavado de mano vigoroso, antes de la manipulación.

- Relación enfermera-paciente 1:1. 
- Vigilar signos y síntomas de infección.

- Extremar las medidas de asepsia y antisepsia.

- Realizar una adecuada desinfección a las conexiones del ventilador, con la solución antiséptica disponible y luego esterilizar. (Castro, Gonzalez, \& Alfonso, 2005)

\section{Conclusiones.}

Las consultas por problemas dermatológicos son frecuentes en Pediatría, lo que refuerza la importancia de la formación dermatológica para los profesionales pediátricos, tanto en Atención Primaria como en Atención Hospitalaria, sin olvidar los servicios de urgencias pediátricas. La Atención Primaria se constituye, por su accesibilidad y proximidad a la población, en una vía sencilla y habitual para la consulta de los problemas dermatológicos pediátricos, y es el pediatra, por consiguiente, el dispensador de esta atención, que incluye: el enfoque diagnóstico inicial, las normas terapéuticas correspondientes y el seguimiento de los procesos desde la cercanía de su consulta. Asimismo, gracias a una estrecha y fluida relación con el especialista en dermatología, el pediatra propone y gestiona la consulta con éste cuando el caso así lo aconseja, evitando, de esta manera, las derivaciones innecesarias. La dermatitis atópica, las infecciones cutáneas y las zoonosis, que serían los motivos de consulta dermatológica más frecuentes, la mayoría de procesos dermatológicos de la infancia pueden ser manejados en Atención Primaria, manteniendo una estrecha colaboración con los especialistas en dermatología para aquellas situaciones que el escenario clínico así lo precise. (Menéndez Tuñón, Sariego Jamardo, Fernández Tejada, Fernández García, López Vilar, \& Meana Meana, 2010) 
Se concluye que definitivamente debe dirigirse la atención al paciente pediátrico, en el sentido de las medidas preventivas y de higiene, así como el cuidado adecuado de la piel. Por otra parte, el alto porcentaje de pacientes en edad pediátrica destaca la necesidad del desarrollo de una consulta de dermatología pediátrica en los Post grados de Dermatología a cargo de un equipo de dermatólogos con capacitación en esta área, quienes a su vez adiestren a los médicos residentes en formación, en lo que será gran parte de su consulta como Especialistas en Dermatología. (Roye, Meléndez, Ruíz, Gamboa, \& Morantes, 2006)

Una lesión en la piel puede ser puerta de entrada para microorganismos, potenciando la morbilidad, y aún la mortalidad en ésta primera etapa de la vida. Es importante considerar la trascendencia de éste órgano como barrera contra las infecciones, protección de los órganos internos, colaborador en el mantenimiento de la temperatura y regulador de agua y electrolitos. La piel es también un órgano sensorial, que facilita el contacto inicial, la relación entre él y su madre. Las sensaciones táctiles de dolor, de temperatura, de presión son recibidas a través de la piel y transmitidas al cerebro. Las enfermeras que cuidan recién nacidos prematuros utilizan la piel para calcular su madurez, valorar la edad gestacional, puntuar el grado de adecuación a la vida extrauterina, y determinar su vitalidad. El cuidado inadecuado de la piel es una causa de morbilidad en los recién nacidos prematuros. La prevención es fundamental para el cuidado de estos niños vulnerables. Reducir al mínimo las lesiones debe ser considerado un componente clave en el cuidado diario brindado a los recién nacidos prematuros. Las enfermeras tienen un desafio: someter bajo la evidencia las nuevas intervenciones de enfermería y productos para el cuidado de la piel del recién nacido, también, apoyar las nuevas investigaciones sobre prácticas de cuidado de la piel, que puedan generar mejores cuidados para los recién nacidos. (Chattás, 2010) 
La prevención de la sepsis neonatal comienza con la prevención del parto prematuro. Por esto, se deben dirigir esfuerzos en colaborar como parte de un equipo multidisciplinario asegurando el cumplimiento de las estrategias de prevención. Es necesario incrementar la búsqueda prenatal de estreptococo del grupo B (SGB) mediante el fortalecimiento de las metas asistenciales y de la adherencia a los protocolos de atención en el control prenatal en nuestra población, así como mejorar el rendimiento de las técnicas diagnósticas utilizadas al momento del parto y lograr la tipificación de las cepas aisladas, con miras a optimizar las acciones terapéuticas. El conocimiento local de la epidemiología y su forma de presentación es fundamental para diseñar estrategias que, en conjunto, contribuyan a un descenso significativo de la incidencia, morbilidad y mortalidad, y que a su vez permitan optimizar recursos y disminuir los costos de salud asociados a esta enfermedad. (Herrera, y otros, 2018)

Es fundamental el manejo nutricional con aporte rico en calorías y proteínas necesarias, las cuales pueden ayudar a la recuperación en pacientes. Debe haber acompañamiento por parte de pediatría, dermatología, fisioterapia, ortopedia, cirugía plástica, cirugía pediátrica, nutrición, odontología, psiquiatría y psicología entre otras (Torres, Contreras, \& Gonzalez, 2011)

Finalmente tenemos unas Normas generales de higiene y epidemiología en los departamentos de neonatología para prevenir infección:

- El acceso al departamento debe ser limitado.

- Lavado de mano higiénico antes de entrar al departamento.

- Cumplir estrictamente el flujograma del departamento.

- Uso correcto de las soluciones desinfectantes disponible. 
- Uso correcto del porte personal (evitar las prendas, pelo recogido, ropa adecuada).

- Evitar el hacinamiento en la sala y de personas en los cubículos.

- Las unidades de neonatología deben tener una buena climatización.

- El material estéril bebe estar bien protegido y durar el tiempo reglamentado.

- Cumplir las normas del uso y desinfección del material estéril.

- Mantener una buena higiene en el departamento.

- Cumplir las normas de aislamiento en un paciente séptico.

- Llevar un control estricto de los cultivos y perfiles de sepsis de los pacientes.

- Acortar la estadía de los neonatos en el departamento, según sus posibilidades.

- No usar indebidamente los antibióticos para evitar de esta forma la resistencia antimicrobiana (Castro, Gonzalez, \& Alfonso, 2005).

\section{Bibliografia.}

Bolognia, J., Schaffer, J., \& Cerroni, L. (2018). Dermatología. Cuarta Edicion. Barcelona: Elsevier.

Castro, F. W., Gonzalez, G., \& Alfonso, J. E. (2005). Cuidados de enfermería para la prevención de las infecciones posnatales. Revista Cubana de Enfermería, ISSN 0864-0319. .

Chattás, G. (2010). Cuidados de la piel del recién nacido pretérmino. Revista de Enfermeria, 10-17.

Coronell, W., Pérez, C., Guerrero, C., \& Bustamante, H. (2009). Sepsis neonatal. Revista de Enfermedades Infecciosas en Pediatría Vol. XXIII Núm. 90, 57-68.

Cutrone, M., \& Peruzzetto, C. (2006). Lesiones iatrogénicas en la piel del recién nacido. Dermatologia pediatrica Latinoamericana, 9-18. 
Gómez, M., Danglot, C., \& Aceves, M. (2012). Clasificación de los niños recién nacidos. Revista Mexicana de Pediatría, 32-39.

Hernandez, M., \& García, E. (2006). Riesgo de infección intrahospitalaria en la unidad de cuidados neonatales. Revista Electrónica de las Ciencias Médicas en Cienfuegos, 19-24.

Herrera, T., Murillo, M., Gesuele, J., Morales, M., Mota, M. G., Adriana, V., y otros. (2018). Incidencia de sepsis precoz por Streptococcus agalactiae en recién nacidos del Centro Hospitalario Pereira Rossell en el período 2007-2015. Revista chilena de infectología, http://dx.doi.org/10.4067/s0716-10182018000400424 .

Martínez, R., Pastrana, F., Ramirez, C., Naranjo, M., \& Ortiz, P. (1999). Morbilidad por afecciones dermatológicas. estudio estadístico territorial. Revista Cubana de Pediatría, 53-59.

Menéndez Tuñón, S., Sariego Jamardo, A., Fernández Tejada, E., Fernández García, N., López Vilar, P., \& Meana Meana, A. (2010). Consultas dermatológicas en Pediatría de Atención Primaria. Pediatría Atención Primaria, 12(45), 41-52.

Roye, R., Meléndez, M., Ruíz, G., Gamboa, A., \& Morantes, J. (2006). Enfermedades dermatológicas en la enfermedad pediátrica. Hospital Militar "Dr. Carlos Arvelo" 20052006, Caracas Venezuela. Dermatología Venezolana Vol. 44, $N^{\circ} 4,12-16$.

Torres, M., Contreras, C., \& Gonzalez, M. (2011). Epidermólisis ampollosa en un recien nacido, reporte de un caso. Revista CES Medicina (25) $N^{\circ}$ 2, 221-230. 